\title{
Physical Activity Prescription Among Healthcare Professionals in Hospital Setting: In the Application of the Theory of Planned Behavior
}

\author{
Getu Teferi \\ Department of Sports Science, Debremarkos University, Debremarkos, Ethiopia
}

Email address:

teferigetu36@gmail.com

\section{To cite this article:}

Getu Teferi. Physical Activity Prescription Among Healthcare Professionals in Hospital Setting: In the Application of the Theory of Planned Behavior. International Journal of Science, Technology and Society. Vol. 8, No. 1, 2020, pp. 1-8. doi: 10.11648/j.ijsts.20200801.11

Received: February 8, 2019; Accepted: December 20, 2019; Published: January 4, 2020

\begin{abstract}
Introduction: Regular physical activity can achieve greater effects on non-communicable diseases (NCDs) than those achieved with drugs. The healthcare setting has been recognized as an appropriate and promising venue for prescribing physical activity. Aim of the study: To assess physical activity prescription behavior (PAPB) of Ethiopian healthcare professionals and determine if the theory of planned behavior explain this behavior. Methods: A cross-sectional survey design was used to assess healthcare professionals' physical activity counseling and prescription behavior for non-communicable diseases (NCDs) in hospital setting. The survey includes self-reported physical activity prescription behavior, and the tenets of theory of planned behavior (attitude (ATT), subjective norm (SUN), perceived behavioral control (PBC), and intention (INT) to prescribe physical activity). Structural equation modeling was used to estimate the relationship of theoretical constructs to prescribe physical activity. Amos and SPSS software were applied to estimate the predictive utility of the theory. Results: A total of 442 healthcare professionals from 7 government hospitals in Addis Ababa city participated in the study. From these hospitals 387 healthcare professionals (physicians $=135,34.9 \%$, nurses $=218,56.3 \%$ and physiotherapist $=34,8.8 \%$ ) completed the questionnaire. $21.4 \%$ of healthcare professionals very frequently provide verbal prescription, $5.4 \%$ provide written prescription of physical activity for the purpose of treating and preventing NCDs. The fit of the TPB model was well fit (RMSEA $=0.072$, $\mathrm{CFI}=.926$, TLI $=.910$, GFI $=.902)$. The $\mathrm{PBC}(\beta=.25, \mathrm{p}<.05)$ and INT $(\beta=.17, \mathrm{p}<.05)$ explained physical activity prescription behavior and PBC $(\beta=.39, \mathrm{p}<.05)$, ATT $(\beta=.16, \mathrm{p}<.05)$ explained intention to prescribe physical activity. Conclusion: HCPs's physical activity prescription practice was low. The findings of this study were supported the theory and the TPB have the predictive utility in describing and predicting physical activity prescription behavior among healthcare professionals in hospital setting. The TPB confirmed to be a useful theoretical framework that should be used for understanding healthcare professionals' physical activity prescription behavior in hospital setting. The present study found that perceived behavioral control was the strongest predictor of intention followed by attitude and subjective norm. Also not only for intention it was a strong predictor followed by intention for physical activity prescription behavior and had a direct effects on healthcare professionals' PAP behavior Recommendation: Interventions/strategies focused on improving healthcare professionals' perceived ability to provide physical activity prescription and the willingness or motivation of healthcare professionals to prescribe physical activity for their patients are important. For instance, facilitating fitness centers or gyms in hospitals can encourage healthcare professionals to prescribe physical activity for their patients as well as to make active HCPs.
\end{abstract}

Keywords: Physical Activity Prescription, Structural Equation Modeling,

Non-communicable Diseases and Theory of Planned Behavior

\section{Introduction}

Regular physical activity can achieve parallel or greater effects on non-communicable diseases (NCDs) than those achieved with drugs. [1]. The healthcare setting has been recognized as an appropriate and promising venue for prescribing physical activity [2]. The rapidly growing burden of non-communicable diseases (NCDs) in low- and middle- 
income countries is accelerated by the negative effects of globalization, rapid unplanned urbanization and increasingly sedentary lives [3]. NCDs also affect developing countries like Ethiopia. According to World Health Organization [4] estimation that $34 \%$ of death is because of non-communicable diseases from the total death, with a national cardiovascular disease prevalence of $15 \%$, cancer and chronic obstructive pulmonary disease prevalence of $4 \%$ each, and diabetes mellitus prevalence of $2 \%$. Physical inactivity, or sedentary is considered a major risk factor for a number of adverse health outcomes including obesity, hypertension, cardiovascular disease, diabetes mellitus and all-cause mortality [5]. Physical inactivity is associated with the development of major NCDs such as type 2 diabetes, coronary heart disease, breast cancer and colon cancer [6]. According to NCDs country profile [4] reports that physical inactivity in Ethiopia as a risk factor for non-communicable disease accounts $17.9 \%$.

Levels of physical inactivity are rising in many countries with major implications for the general health of people worldwide and for the prevalence of NCDs [7]. Physical activity counseling and prescription have been discussed extensively for the last decade as a means for preventing or treating NCDs predominantly in healthcare settings. Studies show that the majority of patients listen to their physicians with regard to lifestyle behavior guidance, such as diet and physical activity. Still, commitment to follow physical activity advice, provide physical activity recommendation and the motivation to comply with physical activity interventions are major challenges faced by the patient and the healthcare provider [8]. The hospital setting may be a key to achieve the goal of establishing PA as a regular treatment modality for many of the lifestyle related risk factors and disorders [9]. For people with chronic conditions such as coronary heart disease, diabetes, osteoporosis, arthritis and cancer, the healthcare setting is an important vehicle for promoting physical activity. Strategies for promoting physical activity can involve a range of health care professionals [10].

\subsection{Theoretical Framework of Study}

The theoretical framework for understanding healthcare professionals' physical activity prescription behavior comes from Icek Ajzen's [11]. Theory of Planned Behavior (TPB). According to the theory, an individual's intention to perform a particular behavior is the major factor of actual behavior [12]. Intentions are an indicator of an individual's effort to try to accomplish a goal. Individuals' intention considers three factors. The first factor is how favorable or unfavorable they feel about performing the behavior (also called the attitude towards performing the behavior), the second factor the perceived social pressure to perform the behavior, and lastly the individuals' perception of control over the behavior. Generally, a more favorable attitude, higher social pressure, and higher perception of control would result in a greater intention to perform the identified behavior [12].

The theory of planned behavior is one of the most comprehensive and validated theories in the health and exercise behavior research for understanding and predicting exercise behavior, and thus, it has guided much of the exercise behavior research [13], and [14]. The theory of planned behavior is the most important theory to predict/explain the given behavior. Regarding to this a study reports that TPB explain healthcare professionals' (physicians, nurses, pharmacist, and other clinicians) behavior including prescription, referral, counseling, pain management, providing care to patient and guideline adherence [15].

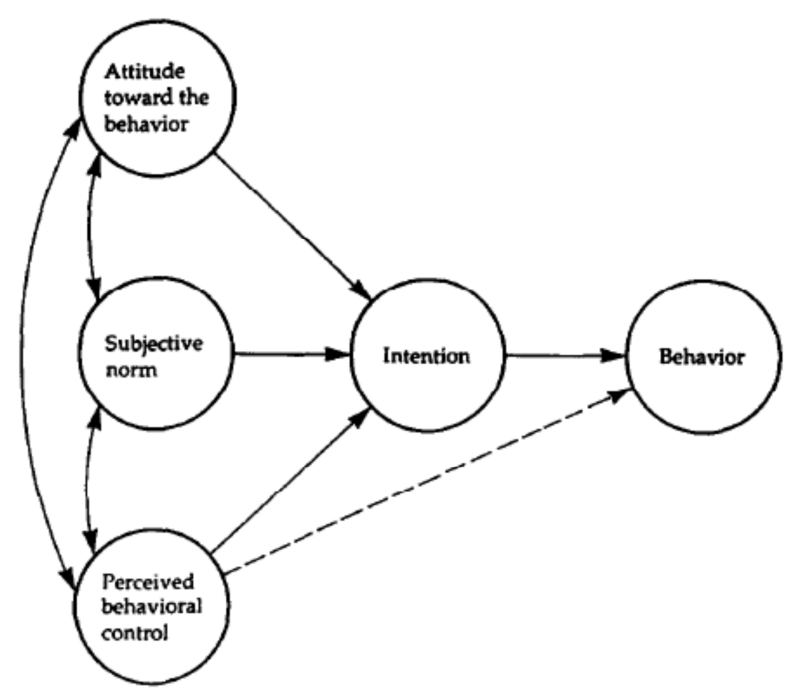

Figure 1. The Theory of Planned Behaviour (Ajzen, 1991).

\subsection{Statement of the Problem}

The Theory of Planned Behavior is useful to predict physical activity behavior among healthcare professionals. Future studies should use this theory to design, assess, evaluate and predict physical activity prescription behaviour and its socio-cognitive determinants (Bardus and Suggs, 2012). Information about healthcare professionals' PA prescription behavior from developing countries especially in Africa is lacking. In Ethiopia, these practices are unknown. Furthermore, the utility of the TPB to explain healthcare professionals' PA prescription behavior remains unknown. Studies are needed to better understand the physical activity prescription behavior among Ethiopian healthcare professionals and the usefulness of the TPB. This information is critical as a potential foundation for future interventions, to prepare physical activity prescription guideline for healthcare professionals as well as persons with chronic diseases, use as initial bases for policy makers, researchers, academicians and who want to establish strategies through physical activity to prevent and manage disease, especially for non- communicable diseases and it helps to promote physical activity/ exercise is medicine in hospital setting as well as in population relevant to Ethiopia. Therefore, the purpose of this study was to describe the PA prescription behavior of healthcare professionals and to determine if the tenets of the TPB explain this behavior.

\subsection{Aim of Study}

Therefore, the overall aim of this study is to test the theory of planned behavior and investigates its predictive utility of 
healthcare professionals' physical activity prescription behavior in hospital setting.

Research Objectives

RO1: To identify the most determinant factors that affects HCP PAPB from TPB

RO2: Assessing Self-reported personal physical activity level of healthcare professionals

\subsection{Research Questions}

RQ1: Is the theory of planned behavior a good predictor of healthcare professionals' physical activity prescription behavior in hospital setting?

RQ2: What was the relationship between perceived behavioral control beliefs and the intention to prescribe physical activity among healthcare professionals in hospital setting?

RQ3: Did healthcare professional's physical activity prescription practice correlate with report of their physical activity level?

\section{Research Method}

A cross-sectional survey design was used to assess Addis Ababa's, healthcare professionals' physical activity counseling and prescription behavior for non-communicable diseases in hospital setting. Ethiopia has a total of 9 regions and two administrative cities. Addis Ababa city is among those and the capital city of Ethiopia. Addis Ababa city was providing the setting for the study. Source population of the study was the registered healthcare professionals (medical doctors, nurses and physiotherapist) with any age and sex and currently on practice in the sample hospitals.

\subsection{Sample and Sampling Procedure}

Participants had to meet the following criteria to include in the study: a registered medical doctors, nurses and physiotherapist, male or female, currently working in the sample hospitals, volunteer and any age. Hospitals that give services related to: cardiovascular diseases, diabetic type 2 and chronic respiratory diseases. First, the researcher was contacted Addis Ababa city health bureau to get information and list of government hospitals. Then based on the information and the inclusion criteria the researcher was selecting the sample hospitals. Second, contact each sample hospitals' medical director and get permission. Finally, by using simple random technique and stratified sampling (for nurses, medical doctors and physiotherapist), from the total of 12 government hospitals 7 hospitals and 442 healthcare professionals as a sample were selected. The sample hospitals were including: Black lion referral hospital, Federal police referral hospital, St. Paulos referral hospital, St. Petere's hospital, Zewditu hospital, Yekatit 12 hospital, and Trunesh Bejing General Hospital.

The sample size was determined by using the formula for estimating a single population proportion. Sample size was calculated by taking the proportion of physical activity prescription/counseling which is $50 \%$ on healthcare professionals (medical doctors, nurses and physiotherapist) for chronic disease with $95 \%$ confidence level, $5 \%$ margin of error to get an optimum sample size that allowed the study to look into various aspects of physical activity prescription/ counseling among healthcare professionals. Based on the above assumptions, the formula is as follows [16]:

$$
\mathrm{s}=\mathrm{X}^{2} \mathrm{NP}(1-\mathrm{P}) \div \mathrm{d}^{2}(\mathrm{~N}-1)+\mathrm{X}^{2} \mathrm{P}(1-\mathrm{P})
$$

$\mathrm{S}=$ required sample size

$\mathrm{X}^{2}=$ the table value of chi-square for 1 degree of freedom at the desired confidence level (3.841) --------------- 1.96 $\mathrm{x} 1.96=3.8416$

$\mathrm{N}=$ the population size

$\mathrm{P}=$ the population proportion (assumed to be.50 since this would provide the maximum sample size).

$\mathrm{d}=$ the degree of accuracy expressed as a proportion (.05).

Based on this formula the maximum sample size was 384 , assume $85 \%$ would be return rate, then add $15 \%$, the total sample have been 442 .

\subsection{Measures and Data Collection Instruments}

Demographic information including physician' sex, age, years of practice, profession and the key variables were collected through questionnaire. Healthcare professional's psychosocial physical activity prescription behavior was measured by questionnaires adapted from a validated theory of planned behavior (TPB) questionnaire [17] and [18]. The questionnaire had four scales, each measuring one construct of the TPB, with answers anchored on a six-point scale. Physicians' attitude toward physical activity prescription was measured with four items, subjective norm was measured with three items, perceived behavioral control was measured with five items, intention to prescribe physical activity was measured with three items and healthcare professionals PA prescription behavior measured with two items. The mean of these items for each scale was used as an indicator of each construct. An average internal consistency of $\alpha=.87$ for the four scales has been previously reported (Ramsay et al, 2010 as cited in [19]. and the $\alpha=.80$ [19]. The internal consistency for the attitude scale was $\alpha=0.95$, for subjective norm $\alpha=$ 0.60 , for perceived behavioral control $\alpha=0.71$, and for intention $\alpha=0.95$ [19]. Healthcare professional's physical activity level was measured from the International Physical Activity Questionnaire (IPAQ) short version survey [20].

\subsection{Data Analysis}

\subsubsection{Statistical Analysis and Considerations}

The analysis for this study involved a preliminary analysis of the main variables (healthcare professionals' physical activity prescription behavior and TPB constructs). Several assumptions should meet before the main analysis performed in order to make generalizations for our findings from structural equation modeling. Data examination is necessary in an initial step in any analysis that the researchers often overlook, the researchers should evaluate the impact of the missing data, identify the outliers and tests for the 
assumption underlying most structural equation modeling techniques [21]. The preliminary analysis of our study shows that: our sample reasonably confirm that what could be expected regarding to outliers, and missing data in cases and variables, regarding to normality, our study indicates that all variables approximately normally distributed, the kurtosis and skewness value of the variables that found between the range of $(-1.96$ to +1.96$)$, the correlation between the predictor variables should not have too high correlation, if the variables have too high correlations, there would be an issue of multicollinearity. Means it measures the same thing, in our study this was not an issue.

After having evaluated the data the relationship among the TPB tenets were run by using structural equation modeling (SEM) techniques. Structural equation modeling (SEM) is a statistical methodology that takes a confirmatory (i.e., hypothesis-testing) approach to the analysis of a structural theory bearing on some phenomenon [23]. Structural equation modeling is a family of statistical techniques and procedures used for testing and estimating causal relationship between and among variables. Data were analyzed by using Amos software packages version 21 for SEM. SEM computer program such as Amos use full information estimation approaches to estimate path coefficients and standard errors simultaneously.

The main analysis of this study was analyzed by using: descriptive statistics were computed to explore the data; structural equation modeling was employed to test the fit of the TPB model using the maximum likelihood method. Path analysis by using Amos software was performed to test the predictive utility of the theory and relationships among the variables.

\subsubsection{Overall Model Fit Criteria}

Table 1. Model Fit Criterion and Acceptable Fit Interpretation.

\begin{tabular}{lll}
\hline Model Fit Criterion & Acceptable Level & Interpretation \\
\hline Chi-square & Not significant, $>0.05$ & Compares obtained chi-square value with tabled value for given df \\
Goodness of Fit Index (GFI) & 0 to 1 & Values close.90 and above reflect good fit \\
Root-mean square error of approximation (RMSEA) & .05 to.08 & Values of.05 to.08 indicates a good model fit \\
Normed fit index (NFI) & 0 to 1 & Values close to.90 or above reflects a good model fit \\
Parsimony fit index ( PNFI) & & \\
Tucker-Lewis index (TLI) & 0 to 1 & Values close to.90 or above reflects a good model fit \\
Comparative Fit Index (CFI) & 0 to 1 & Values close to.90 or above reflects a good model fit \\
CMIN/DF & $3: 1$ & The ratio of $x^{2}$ to the degrees of freedom for a model, 3:1 or less is \\
\hline
\end{tabular}

Source: Schumackerans and Lomax, 2010 and Hair et al, 2010.

\section{Results}

A total of 442 healthcare professionals from 7 government hospitals in Addis Ababa city participated in the study. From these hospitals 387 healthcare professionals (physicians $=135,34.9 \%, \quad$ nurses $=218,56.3 \% \quad$ and physiotherapist $=34,8.8 \%$ ) completed the questionnaire. $21.4 \%$ of healthcare professionals reported that they very frequently provide verbal physical activity prescription for their patients and $5.4 \%$ indicates that they very frequently provide written physical activity prescription for their patients. From the international physical activity questionnaire, healthcare professionals' physical activity level reported that classified as low level $(91,23.5 \%)$, moderate level $(285,73.6 \%)$, and high level $(11,2.8 \%)$. More than half of healthcare professionals $(76.4 \%)$ were classified as physically active. Generally, healthcare professionals had a positive attitude, subjective norm (experienced social pressure) and had relatively moderate perceived behavioral control (perceived their ability to prescribe physical activity but relatively low intention to prescribe physical activity for their patients. Regarding to the relationship between HCPs' physical activity level (PAL) and their prescription behavior, there was no a significant correlation between their PAL and PAPB $(\mathrm{rho}=.061, \mathrm{p}=.231)$.
Table 2. Healthcare Professionals Sample Characteristics.

\begin{tabular}{ll}
\hline Variables & Statistical proportions \\
\hline Demographic variables & \\
Age (m, SD) & $(32,6.5)$ \\
Male (n, \%) & $(177,45.7)$ \\
Women (n,\% & $(210,54.3)$ \\
Work experiences (n, \%) & \\
0-5 years & $(151,39)$ \\
6-10 years & $(108,27.9)$ \\
11-15 years & $(90,23.3)$ \\
Above 15 years & $(38,9.8)$ \\
Healthcare providers profession/department (n, \%) & \\
Physicians (Medical Doctors) & $(135,34.9)$ \\
Nurses & $(218,56.3)$ \\
Physiotherapist & $(34,8.8)$ \\
Physical activity prescription behaviour (Mean, SD \\
Provide physical activity verbal prescription & $(2.57,1.57)$ \\
Provide physical activity written Prescription & $(1.92,1.3)$ \\
Healthcare professionals physical activity level & \\
IPAQs score (Mean, SD) & $(1328.1,821.37)$ \\
Classified as low level (n, \%) & $(91,23.5)$ \\
Classified as Moderate Level (n, \%) & $(285,73.6)$ \\
Classified as High level (n,\% ) & $(11,2.8)$ \\
Theory planned behavior constructs (Mean, SD) & $(4.69,8)$ \\
Attitude & $(4.16,86)$ \\
Subjective norm & $(4.2,8)$ \\
Perceived behavioral control & $(3.9,89)$ \\
Intention & \\
\hline & \\
&
\end{tabular}

Based on the fit indices and guidelines set for this study 
(see Table 1) GFI >.90, RMSEA and RMR between.05 to.08, CFI $>.90$, TLI $>.90$. The fit of the TPB model was well fit. The RMSEA $=.072$ and $\mathrm{RMR}=.076$ indicates that the predicted theory of planned behavior model was consistent with the observed model. The CFI $=.926$, TLI $=.91$ and $\mathrm{CMIN} / \mathrm{DF}=3: 1$ values obtained indicates that the proposed model was better than the null model.

Table 3. Model fit test result.

\begin{tabular}{|c|c|c|}
\hline Model Fit Criterion & Model Fit Result & Decision \\
\hline Chi-square $(>.05)$ & 00 (significant) & Not accepted \\
\hline Goodness of Fit Index (GFI>.90) & 902 & Accepted \\
\hline $\begin{array}{l}\text { Root mean square residual } \\
(\mathrm{RMR}, .05-.08)\end{array}$ & 076 & Accepted \\
\hline $\begin{array}{l}\text { Root-mean square error of } \\
\text { approximation (RMSEA } \\
\text { between.05-.08) }\end{array}$ & 072 & Accepted \\
\hline Normed fit index (NFI>.90) & 894 & Tolerable \\
\hline
\end{tabular}

\begin{tabular}{lll}
\hline Model Fit Criterion & Model Fit Result & Decision \\
\hline & & accepted \\
Tucker-Lewis index $(\mathrm{TLI}>90)$ & 910 & Accepted \\
Comparative Fit Index $(\mathrm{CFI}>.90)$ & 926 & Accepted \\
CMIN/DF $<3: 1$ or $3: 1$ & $3.004: 1 \sim 3: 1$ & Accepted \\
\hline
\end{tabular}

The factor loading for each constructs from the confirmatory factor analysis (CFA) loaded satisfactorily onto their corresponding latent variables (factor loading ranging from.65 to.97). This value indicates that relatively high factor loading, this loading size confirm that indicators are strongly related to their associated constructs. The model measurements of validity and reliability from AVE, CR and Cronbach's alpha have shown that very well fit. The value found that (AVE above.5, CR above.7 and Cronbach's alpha above.8) indicates the model have good validity and reliability (see Table 4).

Table 4. Model Measurement validity and reliability test results.

\begin{tabular}{lllll}
\hline Scales & AVE & CR & Cronbach's alpha & Criteria \\
\hline SBP & 63 & 70 & 833 & \\
ATT & 60 & 76 & 856 & Avecision \\
INT & 58 & 76 & 815 & Accepted \\
PAP & 78 & 64 & 872 & Accepted \\
PBC & 52 & 78 & 840 & Accepted \\
\hline
\end{tabular}

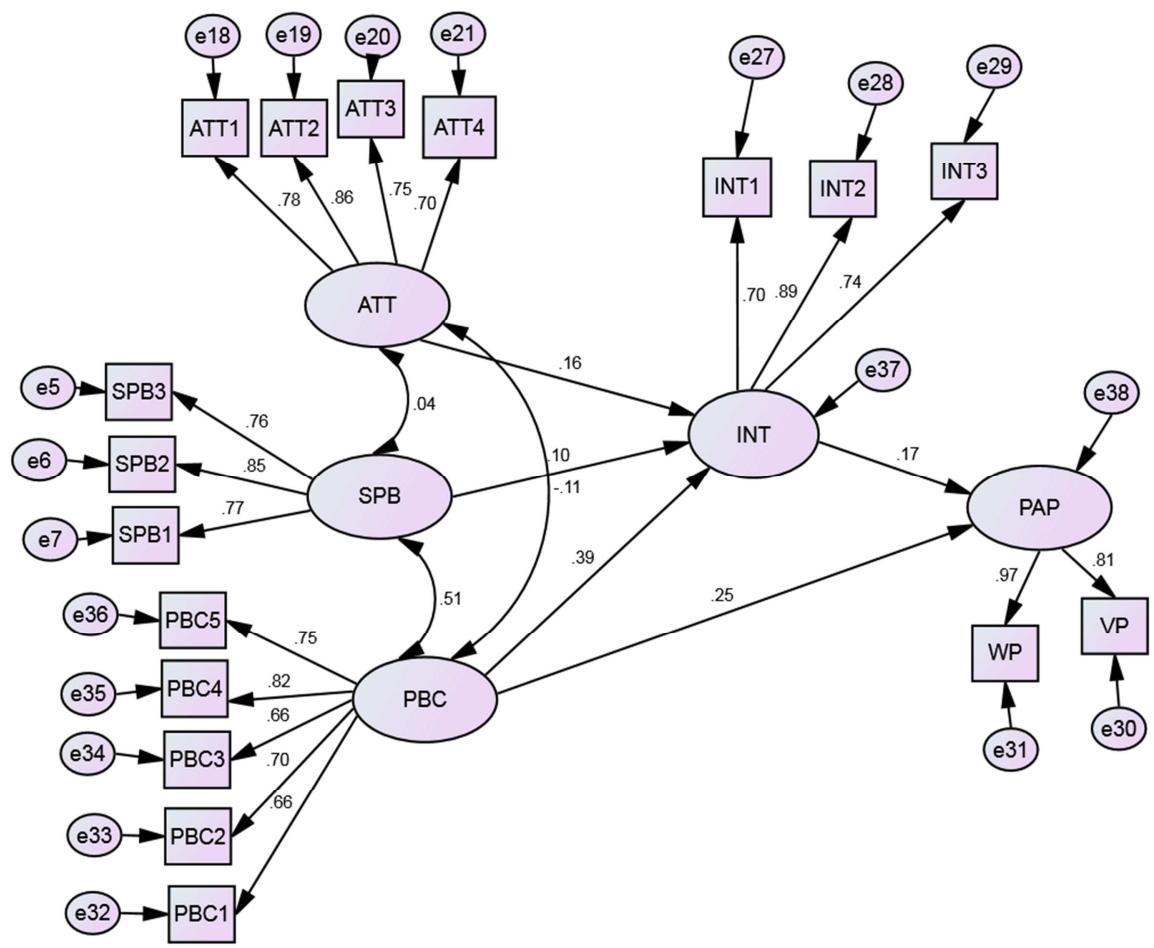

Figure 2. Structural equation model for healthcare professionals' physical activity prescription behavior with standardized parameters.

The structural equation model variables estimates from path analysis indicates that the perceived behavioral control (perceived their ability to prescribe physical activity (PBC) was the strongest predictor of intention $(\beta=.39, \mathrm{p}<.05)$, followed by attitude $(\beta=.16, p<.05)$. Subjective norm (experienced social pressure) $(\beta=.10, \mathrm{p}<.05)$ have relatively a low significant impact on intention to prescribe physical activity. Regarding to physical activity prescription behavior, perceived behavioral control $(\beta=.25, \mathrm{p}<.05)$ and intention to prescribe physical activity for their patients, INT) $(\beta=.17$, $\mathrm{p}<.05)$ explained physical activity prescription behavior. Here INT and PBC were significant predictors of physical activity prescription behavior (see from the path diagram with a standardized parameter estimates is presented in Figure 2). 
Table 5. Correlation of personal physical activity level with physical activity prescription/counseling practices.

\begin{tabular}{llll}
\hline \multirow{2}{*}{ Variable } & \multicolumn{2}{l}{ PALs in MET minutes per week } & \multirow{2}{*}{ P-value } \\
\cline { 2 - 3 } & $\mathbf{N}(\mathbf{3 8 7})$ & $\mathbf{r}_{\mathrm{s}}$ & \\
\hline PAP practice $^{\mathrm{a}}$ & & $164^{* *}$ & 000 \\
\hline
\end{tabular}

The third research question: is that HCPs' personal physical activity level correlate positively and significantly with practices of physical activity prescription/counseling was supported (see Table 5). Personal physical activity level was positively and significantly correlated with physical activity prescription/counseling practices $\left(\mathrm{r}_{\mathrm{s}}=.164, \mathrm{p}=.00\right)$. That means the more physically active the participants, the more frequently prescribe/counsel physical activity to their patients.

\section{Discussion}

The TPB can explain healthcare professional's physical activity prescription behavior; all the theory tenets were supported. The results indicate that perceived their ability to prescribe physical activity (PBC) was the main factor for the behavior of healthcare professionals' intention to prescribe physical activity. PBC and Intention to prescribe physical activity (INT) were the predictors of healthcare professional's physical activity prescription behavior. From these $\mathrm{PBC}$ was the strongest factor to predict this behavior. Their physical activity prescription rates (verbal and written prescription) were low.

Similar study from Mexico, the study of physical activity promotion in Mexican health care setting [19]. show that approximately $48 \%$ of physicians reported that they always ask patients about their physical activity levels, 33\% reported that they always provide verbal physical activity prescription, $6 \%$ indicated that they always provide written physical activity prescription. In our study $21.4 \%$ of Ethiopian, particularly Addis Ababa's healthcare professionals reported that they provide verbal physical activity prescription very frequently to their patients and $5.4 \%$ of healthcare professionals reported that they provide written physical activity prescription very frequently to their patients. In other study which is physical activity counseling and prescription among Canadian primary care physicians investigate that most physicians $(69.8 \%)$ reported using verbal counseling to promote physical activity, whereas only $15.8 \%$ used written prescriptions for a physical activity promotion program.

In our study from the international physical activity questionnaire, healthcare professionals' physical activity level reported that classified as low level (91, 23.5\%), moderate level $(285,73.6 \%)$, and high level $(11,2.8 \%)$. More than half of healthcare professionals $(76.4 \%)$ were classified as physically active. But healthcare professional's physical activity prescription rates for their patients were low, their verbal/written prescription rates were not more than $27 \%$. The study indicates there was no a significant correlation between their PAL and PAPB $(r h o=.061, p=.231)$. When we see other studies the result shows that opposite, healthcare providers own physical activity level influence their physical activity prescription behavior. In other words, a significant relation was found between physical activity counseling and healthcare professionals physical activity level [24] and [25]. In this study we found that the perceived behavioral control (perceived their ability to prescribe physical activity (PBC) was the strongest predictor of intention $(\beta=.39, \mathrm{p}<.05)$, followed by attitude $(\beta=.16, p<.05)$. Subjective norm (experienced social pressure) $(\beta=.10, \mathrm{p}<.05)$ have relatively a low significant impact on intention to prescribe physical activity. According to [19]. completely standardized parameter estimates indicate that subjective norm was the strongest predictor of intention to prescribe PA $(b=0.73, p<$ $0.05)$ followed by attitude $(b=0.16, p<0.05)$. Perceived behavioral control was not a significant predictor of intention $(b=0.04, p>0.05)$. Our study results supported by the theory, "the stronger the intention to engage in a behavior, the more likely should be its performance, the more favorable the attitude and subjective norm toward the behavior, and the greater the perceived behavioral control, the stronger the intention to perform the behavior [11].

Regarding healthcare professionals' physical activity prescription behavior, we found that perceived behavioral control was a significant predictor followed by intention (PBC, $\beta=.25, \mathrm{p}<.05$ ) and INT, $\beta=.17, \mathrm{p}<.05$ ). And intention was positively predicted by attitude, subjective norm and perceived behavioral control. In one study from Nederland [26]. which is health care professionals' intention and behavior to encourage physical activity in patients with cardiovascular risk factors reported that TPB variables accounted for $(\beta=0.41 \mathrm{p}<0.001)$ of the variance in intention. Intention was, in turn, predicted by attitude $(\beta=0.44, p<$ $0.001)$, subjective norm $(\beta=0.201 \%, \mathrm{p}<0.001)$, and perceived behavioral control $(\beta=0.134, \mathrm{p}<0.001)$.

Some limitations should mention to this study. Behavior of healthcare professionals measured by self-report, here our subjects may have over reported their physical activity level and PA prescription behavior. The sampling strategy may be limited, only government hospitals in Addis Ababa were included in this study. Findings are relevant to government hospitals in Addis Ababa Administrative city. This study is the first study in Ethiopia and interpreted with careful and consider as a starting point for other similar future researches.

\section{Conclusion}

This study investigated the use of the theory of planned behavior for predicting the healthcare professionals' physical activity prescription behavior. This investigation had one aim: to test the theory of planned behavior and investigate its predictive utility of healthcare professionals' physical activity prescription behavior in hospital setting. The findings support the predictive utility of TPB in describing and predicting physical activity prescription behavior among Ethiopian healthcare professionals in hospital setting. The TPB confirmed to be a useful theoretical framework that should be used for 
understanding socio-cognitive determinants of healthcare professionals' physical activity prescription behavior in hospital setting. In particular, our study found that perceived behavioral control was the strongest predictor of intention followed by attitude and subjective norm. Also not only for intention it was a strong predictor followed by intention for physical activity prescription behavior and had direct effects on healthcare professionals' PAP behavior. This means that, healthcare professionals with positive and strong control over their own actions were more likely to produce favorable intentions to prescribe physical activity for their patients and can translate the intentions to actions.

Generally, Ethiopian, particularly Addis Ababa's healthcare professionals have a positive attitude towards prescribe physical activity to their patients, experience social pressure to prescribe physical activity was low, and healthcare professionals' intention to prescribe PA to their patients was relatively low but significant. However, Ethiopian, particularly Addis Ababa's HCPs perceive a medium level of ability to prescribe physical activity. But their physical activity prescription rates were low. Because, healthcare professionals' perceived ability to prescribe physical activity, intention to prescribe PA and healthcare professionals' physical activity levels affect this behavior. Strategies which aimed to improve those factors are needed. The problem of low physical activity prescription rate from healthcare professionals in hospital setting requires immediate action and our study investigated the area of improvement which could strengthen efforts conducted in Ethiopian hospital setting.

\section{Recommendations}

Our study indicates the need of some strategies to improve the healthcare professionals' physical activity prescription rates in hospital setting. Healthcare professional's intention to prescribe physical activity was modest not high, so a strategy to improve their intention is important. Ethiopian, particularly Addis Ababa's healthcare professionals perceived ability to prescribe PA needs improvement. Strategies such as short-term training, preparing guidelines and workshops regarding physical activity prescription for non-communicable diseases are important. Finally, a strategy to improve physical activity level of healthcare professionals is important. For instance, facilitating fitness centers or gyms in hospitals can encourage healthcare professionals to prescribe physical activity for their patients as well as to make active HCPs.

\section{References}

[1] Fiuza-Luces, C., Garatachea, N., Berger, N. A., \& Lucia, A. (2013). Exercise is the real polypill. Physiology, 28 (5), 330-358.

[2] Jacobson, D., M., Strohecker, L., Compton, M., T., \& Katz, D., L. (2005). Physical activity counseling in the Aault primary care setting: Position statement of the American College of Preventive Medicine. American Journal of Preventive Medicine, 29 (2): 158-162.
[3] WHO. (2010b). Global Recommendations on Physical Activity for Health. Geneva: World Health Organization.

[4] WHO (2011). The Growing Danger of Non-Communicable Diseases: Acting Now to Reverse Course. Geneva: WHO.

[5] Lee, I. M., Shiroma, E. J., Lobelo, F., Puska, P., Blair, S. N., \& Katzmarzyk, P. T. (2012). Effect of physical inactivity on major non-communicable diseases worldwide: an analysis of burden of disease and life expectancy. Lancet, 380 (9838): 219-29.

[6] Tudor-Locke C, Myers AM. (2001) Challenges and opportunities for measuring physical activity in sedentary adults. Sports Med; 31 (2): 91-100.

[7] World Health Organization. (WHO, 2009). Global Health Risks: Mortality and Burden of Disease attributable to Selected Major Risks (Geneva, WHO).

[8] Daley, A. (2008). Exercise and depression: A review of reviews. Journal of Clinical Psychology in Medical Settings, $15,140-147$.

[9] Borjesson M: Promotion of physical activity in the hospital setting. Dtsch Z Sportmed 64 (2013) 162-165. DOI: 10.5960/dzsm.2012.069.

[10] Milton K, Smith B, Bull F. Action area 3: Health care. In: Blueprint for an active Australia. 2nd edn. Melbourne: National Heart Foundation of Australia, 2014.

[11] Ajzen, I. (1991). The theory of planned behavior. Organizational Behavior and Human Decisions Processes, 50, 179-211.

[12] Ajzen, I. (1998). Models of human social behavior and their application to health psychology. Psychology and Health, 13, 735-739.

[13] Biddle SJH, Nigg CR. Theories of exercise behavior. Int $\mathrm{J}$ Sport Psychol. 2000; 31: 290-304.

[14] Godin G, Kok G: The theory of planned behavior: a review of its applications to health-related behaviors. Am $J$ Health Promot 1996, 11: 87-98.

[15] Godin, G., Belanger-Gravel, A., Eccles, M., \& Grimshaw, J. (2008). Healthcare professionals' intentions and behaviours: A systematic review of studies based on social cognitive theories. Implementation Science, 3 (1), 36.

[16] Krejcie, R. V. \& Morgan, D. W. (1970). Determining sample size for research activities. Educational and Psychological Measurement, 30, 607-610.

[17] Galaviz KI, Jauregui E, Fabrigar L, Latimer-Cheung A, Lopez y Taylor J, Lévesque L. (2014). Physical activity prescription among Mexican physicians: a structural equation analysis of the theory of planned behaviour. International Journal of Clinical Practice (under review).

[18] Ajzen I. (2006). Constructing a TPB questionnaire: Conceptual and methodological considerations. [cited 14 January 2017] Available from: http://www.people.umass.edu/aizen/pdf/tpb.measurement.pdf.

[19] Galaviz KI, Jauregui E, Fabrigar L, Latimer-Cheung A, Lopez y Taylor J, Lévesque L (2015). Physical activity prescription among Mexican physicians: a structural equation analysis of the theory of planned behaviour. International Journal of Clinical Practice, 69 (3) 375-383. 
[20] The International Physical Activity Questionnaire (IPQ, 2005) Guidelines for Data Processing and Analysis of the International Physical Activity Questionnaire (IPAQ) - Short and Long Forms November 2005.

[21] Hair J. F, Black W. C, Babin B. J and Anderon R. E (2010). Multivariate Data Analysis ( $7^{\text {th }}$ ed.). Pearson Prentice Hall.

[22] Hu, L. T. and Bentler, P. M. (1999), "Cutoff Criteria for Fit Indexes in Covariance Structure Analysis: Conventional Criteria Versus New Alternatives," Structural Equation Modeling, 6 (1), 1-55.

[23] Byrne, B. M. (2010). Structural Equation Modeling with AMOS: Basic Concepts, Applications, and Programming (2nd ed.). New York, Routledge Academic.
[24] Ahmed S. A ljaberi (2014). Assessment of Physical Activity (Counseling) at Primary Health Care Centers in Aseer Region, Saudi Ara Med. J. Cairo Univ., Vol. 82, No. 2, September: 207-213, 2014 www.medicaljournalofcairouniversity.net bia,

[25] Johansen KL, Sakkas GK, Doyle J, Shubert T, Dudley RA: Exercise counseling practices among nephrologists caring for patients with dialysis. Am J Kidney Dis 41: 171-178, 2003.

[26] Sassen, B., Kok, G., \& Vanhees, L. (2011). Predictors of healthcare professionals' intention and behaviour to encourage physical activity in patients with cardiovascular risk factors. BMC Public Health, 11 (246). 